\title{
Novel biomarkers associated with kidney function decline
}

Markers of inflammation are associated with an increased risk of cardiovascular disease (CVD); however, the association of these markers with the onset and progression of kidney disease has remained unclear owing to the frequent use of patient cohorts with underlying CVD and chronic kidney disease (CKD). Now, a study published in the American Journal of Kidney Diseases has shown that a decrease in kidney function is indeed associated with biomarkers of inflammation and coagulation.

\section{4 ...biomarkers of} inflammation and coagulation are associated with decreasing kidney function... 77

Hiramoto et al. conducted a 5-year prospective observational study of 4,966 patients from the Multi-Ethnic Study of Atherosclerosis (MESA).
Participants included in the study had a cystatin C-based estimated glomerular filtration rate $(\mathrm{eGFR})>60 \mathrm{ml} / \mathrm{min} / 1.73 \mathrm{~m}^{2}$ and no established CVD at study onset. The researchers determined the associations of C-reactive protein (CRP), IL-6, fibrinogen, factor VIII and D-dimer levels with kidney function decline. A decrease in kidney function was defined as either rapid (eGFR decrease $>3 \mathrm{ml} / \mathrm{min} / 1.73 \mathrm{~m}^{2}$ per year) or incident low eGFR (eGFR $<60 \mathrm{ml} /$ $\mathrm{min} / 1.73 \mathrm{~m}^{2}$ at any follow-up examination and $\mathrm{eGFR}>1 \mathrm{ml} / \mathrm{min} / 1.73 \mathrm{~m}^{2}$ per year).

In linear models, IL-6, a marker of inflammation, and factor VIII, a marker of coagulation, were both independently associated with a decrease in kidney function after adjustment for age, sex, race and comorbid conditions. The researchers found consistent findings using creatinine-based estimations of glomerular filtration rate.

Further analysis revealed that levels of IL-6, factor VIII and CRP were significantly associated with rapid kidney function decrease; only IL-6 was significantly associated with incident low eGFR.

The researchers believe that their findings demonstrate an association between biomarkers of inflammation and coagulation with decreasing kidney function in adult patients without established CVD or CKD and suggest that inflammation or coagulation signaling pathways might contribute to the onset of CKD. They say that further studies are now needed to determine whether suppression of inflammation and coagulation could lead to a reduction in the incidence and progression of CKD.

Katherine Smith 